\title{
ANÁLISES CLIMÁTICAS: O FILTRO HODRICK-PRESCOTT APLICADO AOS ÍNDICES ATMOSFÉRICOS DA OSCILAÇÃO SUL E DA OSCILAÇÃO DO ATLÂNTICO NORTE
}

\author{
ANDERLAN HENRIQUE BATISTA SIQUEIRA E LUIZ CARLOS BALDICERO MOLION
}

\author{
Instituto de Ciências Atmosféricas,Universidade Federal de Alagoas, Maceió, AL \\ anderlansiqueira@gmail.com,lcmolion@gmail.com
}

Recebido Janeiro de 2013 - Aceito Outubro de 2014

\begin{abstract}
RESUMO
A existência de ciclos ou periodicidades, aparentes nas séries temporais longas de variáveis meteorológicas, auxilia no entendimento dos processos físicos que controlam o clima e os ciclos identificados, eventualmente, servem como prognosticadores do clima regional. Com essa motivação, foi aplicado o filtro de Hodrick-Prescott (HP) às séries dos índices de Oscilação Sul (IOS) e Oscilação do Atlântico Norte (OAN) no período 1900-2010. O filtro HP, aplicado a uma série temporal longa, gera duas novas séries, uma contendo os sinais da tendência e a outra, os da parte cíclica/aleatória da variável. Posteriormente, cada uma das séries foi submetida à análise das transformadas de ondeletas de Morlet para identificação dos ciclos aparentes. A série da tendência dos IOS apresentou ciclos de 6, 9, 17 e 28 anos, o primeiro associado ao Ciclo El Niño/La Niña, os dois seguintes possivelmente ao Ciclo Nodal Lunar (Ciclo Saros) de 18 anos e o último, à Oscilação Decadal do Pacífico (ODP). A série da tendência de OAN apresentou ciclos de 6, 9 e 33 anos, os dois últimos possivelmente relacionados ao Ciclo Saros. Os coeficientes de correlação linear entre a série cíclica/aleatória do IOS, adiantado de 6 meses, e a precipitação no Brasil apresentaram valores altos, de até 0,72 e -0,67 para áreas do Norte e Sul do País, respectivamente, no período 1948-1999 (52 anos), com nível de significância superior a 99\%. Esse período cobre as duas fases da ODP. Considerando que os modelos de clima ainda não apresentam destreza adequada para previsão regional no Brasil, sugere-se que a previsão de clima, utilizando a série cíclica/aleatória do IOS adiantado de até 6 meses, seja explorada e, eventualmente, validada.
\end{abstract}

Palavras-chaves: diagnósticos e prognósticos climáticos, ciclos climáticos, ODP.

ABSTRACT: CLIMATE ANALYSIS: THE HODRICK-PRESCOTT FILTER APPLIED TO SOUTHERN OSCILLATION INDEX AND NORTH ATLANTIC OSCILLATION INDEX

The existence of apparent cycles or periodicities in long time series of meteorological variables helps understanding the physical processes that control the climate and the identified cycles may serve as tools for regional climate prognostics. With this insight, the Hodrick-Prescott (HP) filter was applied to the series of Southern Oscillation (SOI) and North Atlantic Oscillation (NAO) indices covering the 1900 to 2010 period. The application of the HP filter to a time series results into two new time series, one of the trend and other of the cyclic/random signal of the variable. Afterwards, the two series were submitted to the Morlet wavelet transform analysis for identifying the apparent cycles. The SOI trend presented cycles of 6, 9, 17 and 28 years, the first associated to the El Niño/La Niña Cycle and the next two possibly related to the 18 year Lunar Nodal Cycle (Saros Cycle), and the remainder, to the Pacific Decadal Oscillation (PDO) cycle. The NAO trend showed 6, 9 and 33 year cycles, the last two possibly related to the Saros Cycle. The linear correlation coefficients between the cyclic/ random SOI series, with 6 month lead, and the Brazilian rainfall totals presented high values, up to 0.72 and -0.67 for the country's northern and southern regions, respectively, during the 1948-1999 (52 years) period, with a statistical significance level above $99 \%$. This period covers the two recent PDO phases. Considering that climate models still do not have still adequate skill for regional forecasting in Brazil, it is suggested that the climate forecasting technique, using up to 6 month leading time of the cyclic/random SOI series, be exploited and, eventually, validated.

Keywords: Climate diagnostics and prognostics, climate cycles, PDO. 


\section{INTRODUÇÃO}

A missão principal da Meteorologia é prever o tempo e o clima. A previsão do tempo, em escala de dias, melhorou sensivelmente nas últimas duas décadas. Porém, a previsão climática, com pelo menos seis meses de antecedência, ainda representa um grande desafio. Como os modelos de clima ainda estão num estágio de desenvolvimento insatisfatório, a ideia que ganhou adeptos ao longo da última década foi a de se utilizar a previsão por similaridade. Esta é uma técnica estatístico-observacional de previsão que avalia o estado atual do clima global e utiliza a análise de dados históricos, índices climáticos deles derivados e suas aparentes periodicidades, na tentativa de identificar, em épocas passadas, estados ou modos climáticos semelhantes ao atual, sob a hipótese que o clima atual e sua variabilidade são semelhantes a um dado modo climático passado.

A dinâmica do clima é condicionada por variabilidades de altas e baixas frequências, algumas periódicas e outras aleatórias, relacionadas com fenômenos naturais que ocorrem fora e dentro do planeta. São exemplos de fenômenos externos periódicos a variabilidade dos parâmetros orbitais terrestres e o Ciclo Nodal Lunar de 18 anos (Ciclo Saros). Esse ciclo, menos conhecido, modifica a distribuição de massas dos oceanos, fazendo com que ocorram mudanças na velocidade das correntes oceânicas que, por sua vez, modificam o transporte de calor e a configuração das temperaturas da superfície do mar (TSM) latitudinalmente (Maksimov e Smirnov, 1965; Yndestad, 2006). Fenômenos externos aleatórios podem ser exemplificados por grandes erupções vulcânicas e a variabilidade da atividade solar. Exemplos de fenômenos internos ao sistema climático na escala global são as variabilidades da cobertura de nuvens, dos transportes de calor sensível e latente, e das TSM. As séries temporais longas de variáveis atmosféricas e oceânicas são resultantes da complexa interação não linear dessas variabilidades. Sendo assim, a separação das baixas e altas frequências torna-se desejável para poder analisar séries longas de dados meteorológicos, seus índices climáticos derivados, e auxiliar no entendimento da variabilidade climática nas variadas escalas de tempo. Hodrick e Prescott (1980) propuseram um método, conhecido como filtro Hodrick-Prescott (HP), que pode auxiliar nesse aspecto, uma análise estatística para tratamento de séries temporais que remove as flutuações de baixa frequência da série original. Ou seja, o filtro HP gera duas novas séries temporais a partir da série original, uma de baixas frequências (tendência) e outra de altas frequências (cíclica/aleatória). O filtro HP não tem sido amplamente utilizado em Meteorologia e este estudo é uma proposição no sentido de avaliar o desempenho desse filtro nas análises de séries temporais de índices climáticos.
A troposfera é aquecida por baixo e, portanto, a condição de contorno inferior mais importante é os oceanos. A interação atmosfera-oceanos, em face da área coberta pelos oceanos e de sua grande capacidade térmica, é um dos processos físicos mais importantes para regular as condições climáticas do planeta. Fenômenos oceânicos são persistentes, devido a sua inércia, e mudam o fornecimento de calor para a atmosfera, modificando, por exemplo, o campo de pressão atmosférica e, por consequência, a circulação geral da atmosfera (CGA). Como a atmosfera não tem limites físicos iguais aos oceanos, essas mudanças do campo de pressão, numa escala global, mudam o campo de ventos e o regime da precipitação pluviométrica em todo o globo por meio de teleconexões. Na atmosfera tropical, se têm dois ramos da CGA, a Circulação de Walker (CW) e Circulação de Hadley $(\mathrm{CH})$. A CW se estende na direção leste - oeste, isto é, zonal, redistribuindo calor dentro da região tropical, e tem seus ramos ascendentes sobre o Pacífico Ocidental (Indonésia), Amazônia e África Equatorial. A CH tem direção equador-trópico, ou seja, meridional, tem a Zona de Convergência Intertropical (ZCIT) como seu ramo de movimentos ascendentes, e é parcialmente responsável pelo transporte do excesso de calor dos trópicos para as regiões subtropicais. O restante é transportado pelas correntes marinhas.

No Oceano Pacífico Tropical, ao sul do equador, existe uma variabilidade no campo de pressão ao nível médio do mar (PNM), com dois centros de ação de polaridade oposta, uma gangorra barométrica decorrente da variabilidade do posicionamento dos ramos ascendentes da CW (Rasmusson e Carpenter, 1982; Trenberth, 1997). Para se caracterizar a intensidade dessa gangorra, foi criado um índice igual à diferença entre as anomalias de PNM em Tahiti (Pacífico Centro-oriental, $17^{\circ} \mathrm{S} ; 150^{\circ} \mathrm{W}$ ) e Darwin (Pacífico Ocidental, $\left.12^{\circ} \mathrm{S} ; 130^{\circ} \mathrm{E}\right)$, ao qual se deu o nome de Índice da Oscilação Sul (IOS). O IOS está relacionado com a ocorrência dos fenômenos El Niño (EN) e La Niña (LN), apresentando, em geral, os valores negativos para eventos EN e positivos para os eventos LN. Uma forma de calcular o IOS foi dada por Troup (1965).

Por sua vez, a troposfera sobre o Oceano Atlântico Norte apresenta uma oscilação, que condiciona o clima da América do Norte e da Europa Ocidental, denominada Oscilação do Atlântico Norte (OAN). Jones et al (1997) calcularam um índice de OAN utilizando as diferenças entre as anomalias de PNM de Lisboa (alta pressão subtropical) e as da Islândia (baixa pressão), enquanto Osborn et al (1999) usaram a diferença entre as de Gibraltar e as da Islândia. A OAN possui duas fases bem definidas, a fase positiva - com a Baixa da Islândia e a Alta Subtropical mais intensas, i.e, PNM abaixo da média de longo prazo na Islândia e acima na Alta Subtropical - e a fase negativa, com a Baixa da Islândia e a Alta Subtropical mais fracas, isto é, PNM acima da média na Islândia e abaixo na Alta Subtropical . 
Objetivamente, utilizou-se o filtro HP nas séries dos índices da Oscilação Sul (OS) e da Oscilação do Atlântico Norte (OAN), para separar os componentes da tendência de prazo longo (que representam oscilações decadais) das variabilidades de prazos curtos (que representam as oscilações interanuais/ sazonais). Para auxiliar na identificação dos ciclos aparentes nas séries que representam tendência e dos ciclos de variabilidade de prazos mais curtos, lançou-se mão da técnica das transformadas de ondeletas de Morlet, que avalia os níveis de energia associados às periodicidades das frequências baixas e altas.

\section{MATERIAIS E MÉTODOS}

Os estudos da variabilidade climática requerem que grandezas físicas e os índices decorrentes sejam medidos continuamente ao longo do tempo. Logo, uma série temporal pode ser definida como sendo uma série de observações representada em função do tempo. A análise de séries temporais é feita utilizando-se um conjunto de técnicas ou métodos estatísticos com o objetivo de identificar possíveis ciclos existentes e úteis para gerar projeções futuras. Existem várias técnicas empregadas em análises de séries temporais. Aqui se optou pelas técnicas filtro Hodrick-Prescott (HP) e transformadas de ondeletas de Morlet.

As séries temporais dos índices da Oscilação Sul (IOS) e da Oscilação do Atlântico Norte (OAN) dispostas na escala mensal foram submetidas às análises por esses métodos. As séries cobrem o período de janeiro de 1900 até dezembro de 2010 e ambas estão disponíveis na página internet do Earth System Research Laboratory, Physical Science Division/ National Oceanic and Atmospheric Administration (ESRL/PSD/ NOAA). As correlações lineares para o território nacional foram elaboradas com dados mensais de precipitação observados, que foram compilados e interpolados em pontos de grade com resolução de $0,5^{\circ} \times 0,5^{\circ}$ para as regiões continentais do mundo pela Universidade de Delaware (UDEL). Os dados de precipitação cobrem o período 1948 a 2010 e estão disponíveis na mesma página, juntamente com a descrição da metodologia empregada em sua compilação e interpolação. Porém, cabe aqui uma advertência, deve-se levar em consideração que tais dados interpolados contém considerável incerteza, tendo em vista a falta de observações em amplas áreas da América do Sul antes da década de 1960.

\subsection{Filtro Hodrick-Prescott}

O filtro Hodrick-Prescott (HP) decompõe uma série temporal em duas séries temporais, uma formada pelos componentes de sua tendência e outra pela parte cíclica/aleatória do sinal original:

$$
y_{t}=\tau_{t}+c_{t}
$$

onde $y_{t}$ é a série original, $\tau_{t}$ e $c_{t}$ são, respectivamente, a tendência e a parte cíclica/aleatória extraídas com o filtro HP. A extração do componente de tendência se dá minimizando a Equação 2, onde $\mathrm{t}=1,2,3 \ldots \mathrm{T}$ :

$$
\min \left\{\sum_{t=1}^{T}\left(y_{t}-\tau_{t}\right)^{2}+\lambda \sum_{t=2}^{T-1}\left[\left(\tau_{t+1}-\tau_{t}\right)-\left(\tau_{t}-\tau_{t-1}\right)\right]^{2}\right\}
$$

O primeiro termo da Equação 2 é a soma da série dos desvios, entre os valores da série original e os respectivos valores da série da tendência, elevados ao quadrado e é uma medida do grau de ajuste. O segundo termo é a soma do quadrado da segunda diferença entre os componentes da tendência e indica seu grau de suavidade. O parâmetro de suavização ou penalização, $\lambda$, controla as variações da taxa de crescimento do componente tendência e deve assumir valores positivos, pois, se $\lambda=0$, a série da tendência seria igual à série original. Por outro lado, quanto maior for $\lambda$, maior é a suavização da tendência, tal que se $\lambda \rightarrow \infty$, a tendência se aproxima de uma linha reta. $\mathrm{O}$ fator crítico na utilização do filtro HP é a escolha dos valores do parâmetro penalizador $(\lambda)$ utilizado na separação do componente da tendência do componente cíclico/aleatório. Hodrick e Prescott (1980) definiram $\lambda$ como sendo:

$$
\lambda_{t}=\left(\frac{\theta c}{\theta \tau}\right)^{2}
$$

onde $\theta c$ é a variância do primeiro termo e $\theta \tau$ a variância do segundo termo da Equação 2. Para dados trimestrais, Hodrick e Prescott (1980) sugeriram $\lambda t=1.600$ baseados, segundo Maravall e Rio (2001), num raciocínio místico pelo qual "em um trimestre, $5 \%$ de desvio no componente cíclico/aleatório é tão moderadamente grande como é $1 / 8$ de $1 \%$ de mudança da taxa de crescimento da tendência", ou seja:

$$
\lambda_{t}=\left(\frac{5}{\frac{1}{8}}\right)^{2}=1.600
$$

Porém, se as frequências de amostragem forem diferentes da trimestral, dados mensais ou anuais, o parâmetro $\lambda$ deve ser ajustado para tais frequências. Ravn e Uhlig (2002) propuseram tratar esse problema no domínio das frequências de amostragem $(\omega)$ que produz resultado generalizado segundo esses autores. Usaram a função de transferência do filtro HP, $h(\omega ; \lambda)$ de King e Rebelo (1993), dada por:

$$
h(\omega ; \lambda)=\frac{4 \lambda[1-\cos (\omega)]^{2}}{1+4 \lambda[1-\cos (\omega)]^{2}}
$$

Esse filtro é semelhante a um filtro passa-alto, em que selecionar valores diferentes para $\lambda$ é comparável a selecionar diferentes valores de frequência de corte do filtro passa-alto. Concluem que: 


$$
\lambda_{\mathrm{s}}=\mathrm{s}^{\mathrm{m}} \lambda_{\mathrm{t}}
$$

onde $\lambda_{\mathrm{t}}$ é o parâmetro trimestral, $\lambda_{\mathrm{s}}$ o parâmetro na frequência $s$ e o expoente $m$ assumiria valores entre 3,8 e 4,0. Para dados de frequência trimestral, adotaram o valor arbitrário de $\lambda_{\mathrm{t}}$ $=1.600$ proposto por Hodrick e Prescott (1980), com $\mathrm{s}=1$. Para dados de frequência mensal $s=3$ e os de frequência anual $s=1 / 4$, substituindo esses valores na Equação (6) com $m=4,0$, obtêm-se $\lambda_{\mathrm{a}}=6,25$ para a frequência anual e $\lambda_{\mathrm{m}}=129.600$ para a frequência mensal.

Na Tabela 1, adaptada de Maravall e Rio (2001), encontram-se os valores dos parâmetros penalizadores a serem utilizados para séries de dados anuais $(\lambda \mathrm{a})$, trimestrais $(\lambda \mathrm{t})$ e mensais $(\lambda \mathrm{m})$ e o correspondente ciclo de referência $(\mathrm{P})$ expresso em anos. O ciclo de referência foi definido como o período de corte em que o filtro utilizado apresenta ganho de $50 \%$. Segundo os autores, a maior parte ciclos superiores (inferiores) ao valor de referência (P) são mantidos na série tendência (cíclica/aleatória). Como os dados utilizados neste trabalho são mensais, optou-se pelo valor $\lambda=14.400$ para filtrar, da série da tendência, os possíveis ciclos existentes com períodos inferiores a aproximadamente 6 anos, como QBO e eventos El Niño.

\subsection{Análise das ondeletas}

Essa técnica estatística foi se desenvolvendo ao longo dos anos e está baseada na transformada de Fourier (TF). Gabor

Tabela 1: Valores dos parâmetros penalizadores para séries de dados anuais $(\lambda \mathrm{a})$, trimestrais $(\lambda \mathrm{t})$ e mensais $(\lambda \mathrm{m})$ e o correspondente ciclo de referência $(\mathrm{P})$ dado em anos.

\begin{tabular}{|c|c|c|c|}
\hline$\left(\lambda_{\mathrm{a}}\right)$ & $\left(\lambda_{\mathrm{t}}\right)$ & $\left(\lambda_{\mathrm{m}}\right)$ & $\mathrm{P}$ \\
\hline & & & $($ anos $)$ \\
\hline 1 & 179 & 14.400 & 5,7 \\
\hline 5 & 1.190 & 95.972 & 9,2 \\
\hline 6 & 1.437 & 115.975 & 9,7 \\
\hline 7 & 1.600 & 129.119 & 9,9 \\
\hline 10 & 2.433 & 196.474 & 11,0 \\
\hline 15 & 3.684 & 297.715 & 12,2 \\
\hline 20 & 4.940 & 399.339 & 13,2 \\
\hline 25 & 6.199 & 501.208 & 13,9 \\
\hline 30 & 7.460 & 603.250 & 14,6 \\
\hline 35 & 8.723 & 705.424 & 15,2 \\
\hline 40 & 9.986 & 807.702 & 15,7 \\
\hline 70 & 17.585 & 1.422 .774 & 18,1 \\
\hline 100 & 25.199 & 2.039 .248 & 19,8 \\
\hline 200 & 50.633 & 4.098 .632 & 23,6 \\
\hline 400 & 101.599 & 8.225 .728 & 28,0 \\
\hline
\end{tabular}

Fonte: adaptada de Maravall e Rio (2001).
(1946) estudou e adaptou a TF para o estudo do sinal no domínio do tempo e no domínio da frequência. Essa modificação ficou conhecida como TF com janela móvel. Porém, existia um grande inconveniente nessa técnica que era o comprimento fixo que tais janelas móveis tinham que ter. Exemplificado, nos estudos feitos na Meteorologia, os fenômenos mudam e apresentam diferenças em escalas de tempo e frequência e, muitas vezes, com ordens de grandezas diferentes para caracterizar um sinal meteorológico.

Morlet et al (1982) e Grossman e Morlet (1984) observaram o estudo feito por Gabor e o modificaram, introduzindo a Análise de Ondeletas (AO). A AO satisfez o Princípio de Heisenberg, introduzindo janelas que se adaptam conforme a frequência em que ocorrem os fenômenos, ou seja, essas janelas se adaptam em relação a fenômenos de baixa ou alta frequência. Weng e Lau (1994) descreveram a AO como um conjunto de procedimentos para análise da variabilidade de séries temporais através de uma série de funções. Essas têm sua gênese por meio de alargamento e translações de uma função simples de variável real qualquer e é conhecida, também, como ondeleta-mãe. Tal função pode ser descrita matematicamente tomando-se uma escala e uma posição, onde o valor da escala tem que assumir valores maiores que zero. A Equação 7 mostra a função da transformada de ondeletas (WY) em uma série continua:

$$
\left(W_{\Psi} f\right)(a, b)=\frac{1}{\sqrt{a}} \int f(t) \Psi\left(\frac{t-b}{a}\right) d t
$$

onde $\mathrm{f}(\mathrm{t})$ é a função que constitui a série de dados a ser analisada, $\Psi=((t-b) / a)$ é a função ondeleta, é utilizado para normalizar a energia de cada ondeleta, "a" é a escala, "b" é a posição e "t" é a variável.

$\mathrm{Na}$ literatura, existem várias funções que são utilizadas para o cálculo e geração das ondeletas (Daubechies, 1992; Foufoula-Georgiou e Kumar, 1994). Torrence e Compo (1998) publicaram um guia prático no qual citam que as funções mais utilizadas são as de Paul, Dog e Morlet. Cada uma possui sua peculiaridade. A utilizada neste estudo foi a função de Morlet. Esta foi escolhida, pois, segundo Weng e Lau (1994), é uma função complexa, que possui características semelhantes as dos sinais meteorológicos que se desejam analisar, tais como simetria ou não, e apresenta uma variação temporal suave ou brusca. A função de Morlet $(\Psi(\mathrm{t}))$ é descrita pela Equação 8:

$$
\Psi(t)=\pi^{-\frac{1}{4}} \cdot e^{i w_{0} t} \cdot e^{-\frac{t^{2}}{2}}
$$

onde " $\mathrm{w}_{0}$ " é a frequência adimensional de Morlet e " $\mathrm{t}$ " é o parâmetro adimensional no tempo.

A técnica de Transformada de Ondeletas (TO) consiste, basicamente, em decompor um sinal em diferentes partes, ou 
seja, explora-se tal sinal em seus níveis de resolução. Esse procedimento é conhecido na literatura como multi-resolução. Ao ser apresentada, a multi-resolução mostra uma figura simplificada para a análise da informação que o sinal traz. As várias resoluções, ou seja, os vários detalhes de um sinal, de forma geral, caracterizam as diferentes estruturas físicas que estão contidas no mesmo. Então, ao modular essa resolução, se podem ressaltar sinais mais fortes ou mais fracos presentes nas séries temporais. Esses sinais são apresentados na forma da intensidade de energia que se representa em função da frequência (período) e da escala temporal da série. Para a geração das figuras, fez-se uso dos softwares livres R/RStudio (software estatístico), pacote mFilter para o filtro HodrickPrescott, e QTOctave (software matemático) para cálculo das ondeletas.

\section{RESULTADOS E DISCUSSÃO}

\subsection{Análise da série do índice da Oscilação Sul}

Na Figura 1, mostram-se os resultados obtidos com o filtro HP para a série dos índices da OS. São vistas a série original dos índices da OS (linha em cor cinza) e da tendência (preto) na Figura 1a e foi representada, na Figura 1b, somente a parte cíclica/aleatória, associada às oscilações de alta frequência.

Observa-se na Figura 1a que a amplitude da linha de tendência (linha em preto) não é acentuada, porém, a oscilação de baixa frequência (períodos longos) se destaca claramente. Nota-se que, em geral, a partir de 1917 e persistindo até 1976, os valores do índice da OS se apresentam predominantemente positivos (modo positivo), à exceção do evento El Niño forte de 1939/1942, se comparados com o do período seguinte, de 1977 a 2005, em que seus valores foram negativos (modo negativo). Esses períodos distintos coincidem, respectivamente, com as recentes fases fria e quente da Oscilação Decadal do Pacífico (ODP), concordando com Molion (2005), que mostrou que os eventos La Niña foram mais frequentes e intensos durante a fase fria da ODP, enquanto os eventos El Niño foram menos frequentes e mais fracos. Durante sua fase quente, ocorre o oposto. É notória, também, a variabilidade interanual, com ciclos de 4 a 5 anos, ocorrendo em cada fase da ODP. Ainda na Figura 1a, durante o período de 1977 a 2005, nota-se que a linha de tendência apresenta amplitude maior e atinge valores positivos em ocasiões em que ocorreram os eventos intensos de La Niña, o de 1988/1989 (seta 1) e o de 1998/2001 (seta 2). Esses eventos mostram que, mesmo num cenário global com a predominância de águas mais quentes (frias) sobre a região do Pacífico Equatorial Centro-Oriental, podem ocorrer IOS positivos (negativos). A correlação entre as séries de IOS e de índice Niño 3.4 mostra um coeficiente de correlação de - 0,63 com nível de confiança de $99 \%$, deixando claro que nem sempre IOS negativos indicam eventos El Niño (EN) e, IOS positivos, eventos de La Niña (LN), como se generaliza na literatura. Na Figura $1 \mathrm{~b}$, nota-se que a parte cíclica/aleatória é tão ruidosa quanto a série original dos índices mensais da OS. Esse fato mostra que a variabilidade do campo de PNM é grande e rápida nas escalas de tempo muito curtas, devido aos fenômenos de tempo de convecção profunda organizada que afetam as

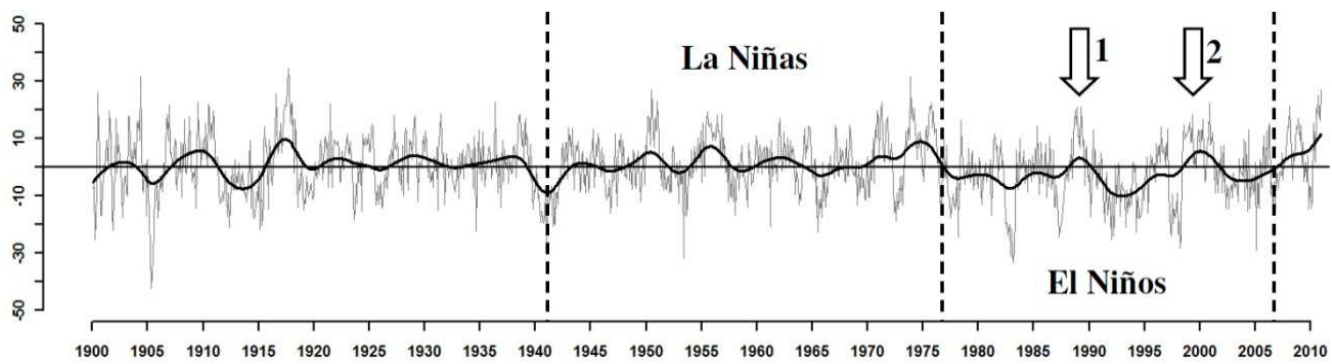

(a)

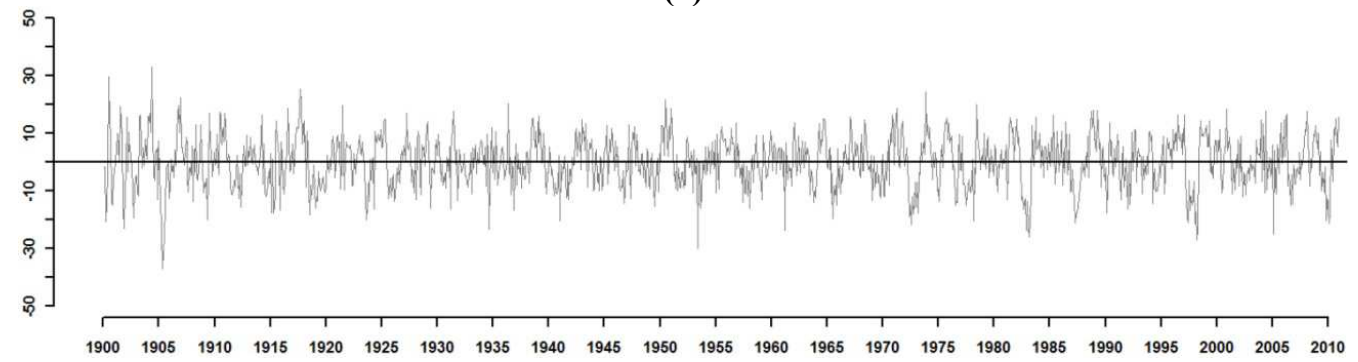

(b)

Figura 1: Análise da Oscilação Sul (OS) em (a) séries dos índices da OS (linha em cor cinza) e da tendência (preto) e em (b) série da parte cíclica/ aleatória, extraídas com o filtro Hodrick-Prescott. 
médias mensais. Ciclones tropicais têm escala sub-mensal e a Oscilação Madden-Julian tem escala sub-sazonal. Portanto, deve-se ter cuidado ao extrapolar os valores mensais de IOS para previsão climática. Notar que as amplitudes dos eventos extremos, como os EN de 1983, 1987 e 1997, são atenuadas, ou filtradas, suavemente e suas magnitudes são semelhantes às da série original do IOS.

Nas Figuras 2a e 2b, mostram-se os resultados das análises das TO aplicadas às séries da tendência e da parte cíclica/aleatória, obtidas com o filtro HP, no período de janeiro de 1900 a dezembro de 2010. As figuras representam, respectivamente, o Espectro de Potência das Ondeletas (EPO), à esquerda, e o Espectro Global das Ondeletas (EGO), à direita. As áreas sombreadas no espectro das ondeletas nas escalas do cinza para o preto são de variâncias normalizadas, as quais estão nas escalas de 0 a 5 unidades com o intervalo de contorno de 0,5 . Os contornos fechados representam nível de confiança superior a $95 \%$ e a curva contínua, em forma de "U", representa o cone de influência, além do qual o efeito de borda se faz importante. No espectro global das ondeletas (EGO), valores são significativos ao nível de $95 \%$ à direita da curva tracejada.

Notam-se dois núcleos de alta energia na Figura 2a, o primeiro de 1910 a 1920 com intervalo no período de 4 a 16 anos e o segundo, de 1966 a 1998, com intervalo no período de 8 a 16 anos. No espectro global das ondeletas (EGO), ainda na Figura 2a, observam-se quatro picos significativos com períodos de anos distintos, um com 6 anos, outro de 9 anos e mais dois com 17 e 28 anos. O espectro apresenta mais energia no final da escala, sugerindo que a oscilação mais forte tenha um período maior que as indicadas. Entretanto, esse final do espectro está fora do cone de influência e não é possível identificar ciclos longos, superiores a 50 anos, com séries temporais relativamente curtas, 110 anos nesta análise. No caso da periodicidade de 6 anos, parece haver uma relação com o Ciclo El Niño/La Niña completo. Ou seja, uma interação oceano-atmosfera direta, águas aquecidas (resfriadas) baixam (aumentam) a PNM em Tahiti e modificam a circulação de Walker. Já os períodos de 9 e 17 anos podem ter relação com o Ciclo Nodal Lunar de 18 anos (Ciclo Saros), que interfere no transporte de calor pelas correntes marinhas em direção aos polos (Maksimov e Smirnov, 1965; Yndestad, 2006) e, posteriormente, mudam o campo de PNM por meio da interação oceano-atmosfera. Maksimov e Smirnov (1965), por exemplo, calculam as variações da inclinação média da superfície do Atlântico Norte entre $45^{\circ} \mathrm{N}$ e $75^{\circ} \mathrm{N}$ para os meses de janeiro e julho no período 1870-1960. Para esses autores, a inclinação da superfície varia de $+6,5 \mathrm{~cm}$ nos anos de máxima declinação lunar e de $-6,5 \mathrm{~cm}$ nos de mínima declinação com relação à declinação média do período estudado. Sugerem, ainda, que essas diferenças de gradiente hidráulico devam influenciar o componente meridional das correntes oceânicas. O período de 28 anos, aparentemente, tem relação com a variabilidade decadal, decorrente da interação entre as fases da ODP e a troposfera sobre esse oceano.

$\mathrm{Na}$ Figura $2 \mathrm{~b}$, série cíclica/aleatória da OS, na qual estão contidos ciclos inferiores a 6 anos inclusive o anual, observam-se picos de alta energia em 2 e 4 anos aproximadamente, com confiabilidade superior ao nível de $95 \%$. Esses podem ter relação direta com a variabilidade associada à Oscilação QuaseBienal (OQB) dos ventos estratosféricos e ao Ciclo El Niño/ La Niña.

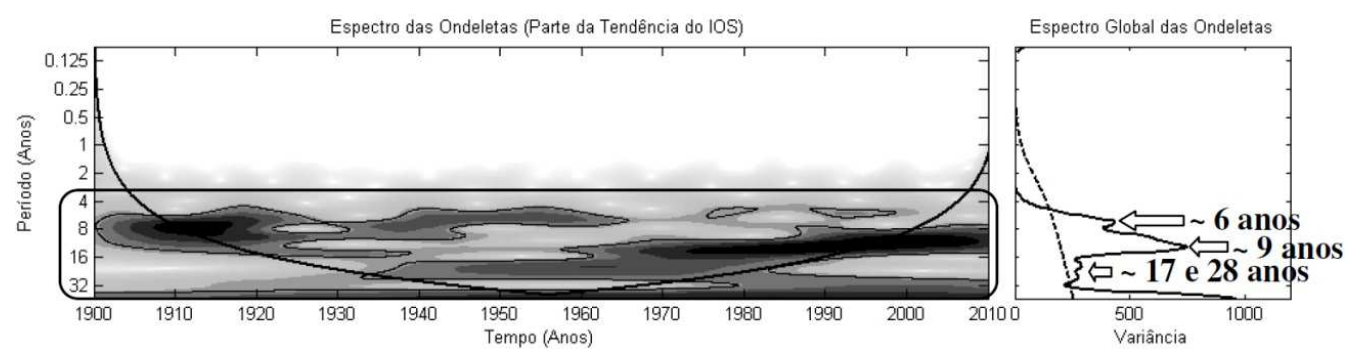

(a)

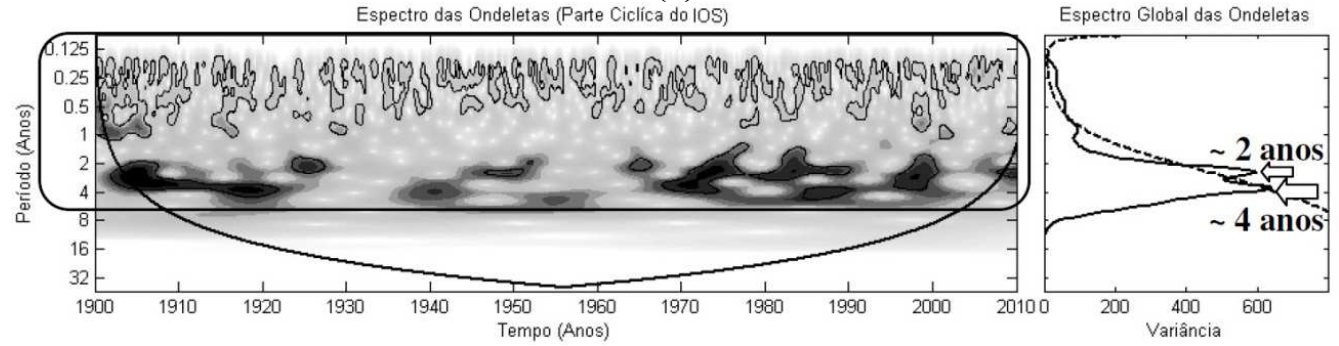

(b)

Figura 2: Análise das ondeletas aplicada às séries (a) da tendência e (b) da parte cíclica/aleatória, obtidas após o tratamento da série dos índices da OS com o filtro HP, com respectivos Espectros de Potências (EPO, esquerda) e Espectro Global das Ondeletas (EGO, direita). 


\subsection{Análise da série do índice da Oscilação do Atlântico Norte}

Na Figura 3, são mostrados os resultados das análises obtidos com o filtro HP para a série dos índices da OAN, que é construída com as anomalias de PNM do Atlântico Norte. Na Figura 3a, apresentam-se os índices da série original da OAN (linha em cor cinza) e a série da tendência (preto) e, na Figura $3 b$, apenas a parte cíclica/aleatória da série desses índices, que corresponde às oscilações de alta frequência.

Não se observam divisões ou modos distintos no índice da OAN (Figura 3a) como observado na série do IOS. Isso concorda com Jones et al (1997) que somente identificaram variações na escala decadal quando restringiram as análises apenas aos meses de inverno boreal. Esse fato parece estar bem estabelecido, pois a variabilidade mais significativa da PNM no Atlântico Norte está associada à Baixa da Islândia, que é característica da circulação de inverno, praticamente desaparecendo no restante do ciclo anual. Na Figura 3b, mostra-se a parte cíclica/aleatória do índice da OAN. Nota-se que não há distinção clara entre a série original do índice (Figura 3a, linha cinza) e a parte cíclica/ aleatória extraída com o filtro HP, de forma geral. Com exceção dos períodos de 1915 a 1920, 1940 a 1945 e de 1988 a 2000, o resultado da análise com o filtro HP indica que o índice da OAN tem variabilidade maior nas escalas de tempo mais curtas, e não apresenta tendência aparente no período analisado.

Nas Figuras 4a e 4b, mostram-se os resultados das análises das ondeletas para as séries da tendência e cíclica/ aleatória, após ter sido aplicado o filtro HP à série temporal dos índices da OAN. Nessas figuras, o Espectro de Potência das Ondeletas (EPO) está à esquerda e o Espectro Global das Ondeletas (EGO) à direita.

$\mathrm{Na}$ Figura 4a, no EPO, identificam-se núcleos de alta energia localizados entre 4 e 8 anos e 8 e 16 anos, o primeiro não-contínuo. A tendência do índice da OAN apresenta 3 picos de energia significativos no EGO de 6, 9 e 33 anos. Os dois primeiros períodos são, surpreendentemente, iguais aos encontrados no espectro do IOS (Figura 2a), embora não necessariamente em fase um com o outro. O período mais longo, de 33 anos, pode estar relacionado à Oscilação Multidecadal do Atlântico (OMA) que, em síntese, é a variabilidade da temperatura da superfície do mar no Atlântico Norte em torno de sua média de longo prazo (Jones et al, 1997). É aceito que o período do ciclo completo da OMA seja cerca de 70 anos. Há sugestões na literatura que o ciclo da OMA seja um múltiplo de Ciclo Nodal Lunar de 18 anos (Yndestad, 2006). Portanto, o período de aproximadamente 33 anos seria um múltiplo desse. Para ambos os resultados, o nível de confiança foi superior a 95\%. Na Figura 4b, parte cíclica/aleatória do índice da OAN que retém os ciclos inferiores a 6 anos inclusive o ciclo anual, observam-se núcleos de alta energia variando entre os períodos de 0,5 ano (6 meses) a 1 ano, que também estão aparentes no EGO ao nível de confiança igual ou superior a 95\%. Esse fato pode estar relacionado ao campo de PNM das regiões, em que são tomados os dados para compor os índices mensais, serem afetadas constantemente por fenômenos transientes de escala de tempo menor, possivelmente, mudanças na amplitude e no número de ondas de Rossby e bloqueios atmosféricos.

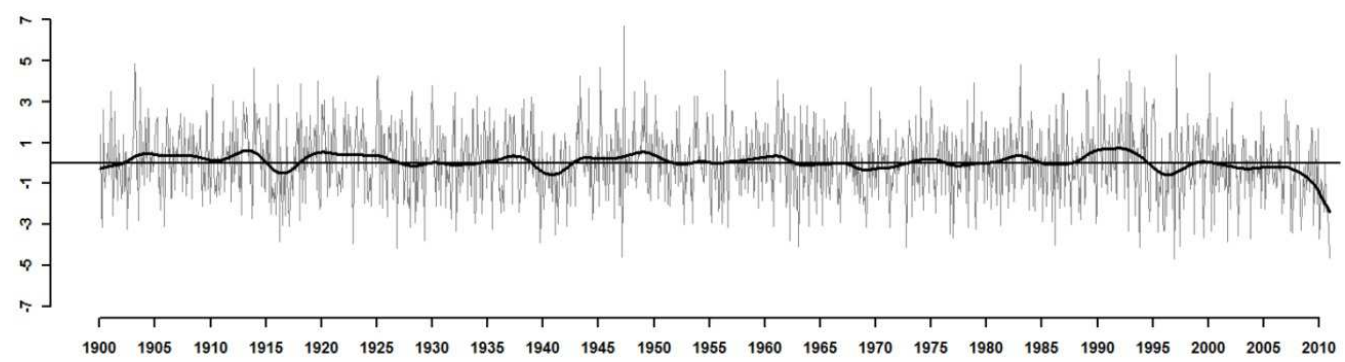

(a)

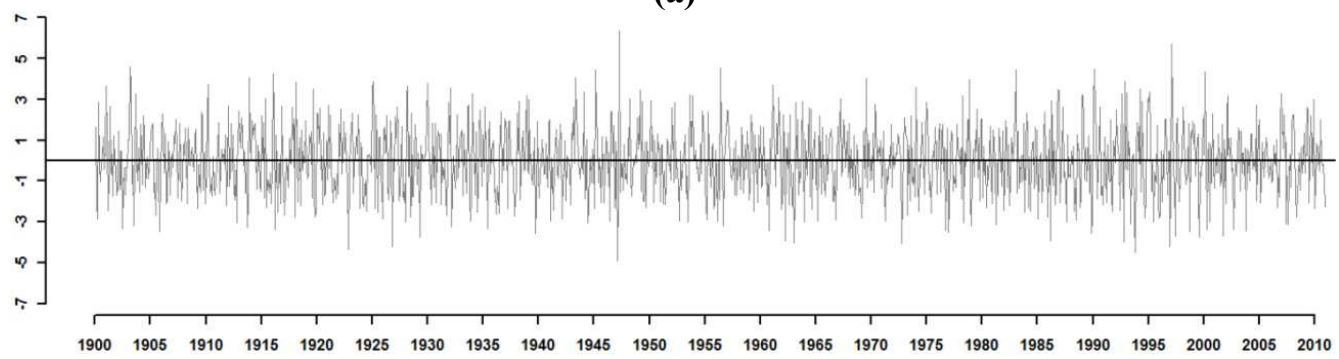

(b)

Figura 3: Análise da Oscilação do Atlântico Norte (OAN) em (a) série dos índices da OAN (linha em cor cinza) e da tendência (em preto) e em (b) a parte cíclica/aleatória, extraídas com o filtro Hodrick-Prescott. 


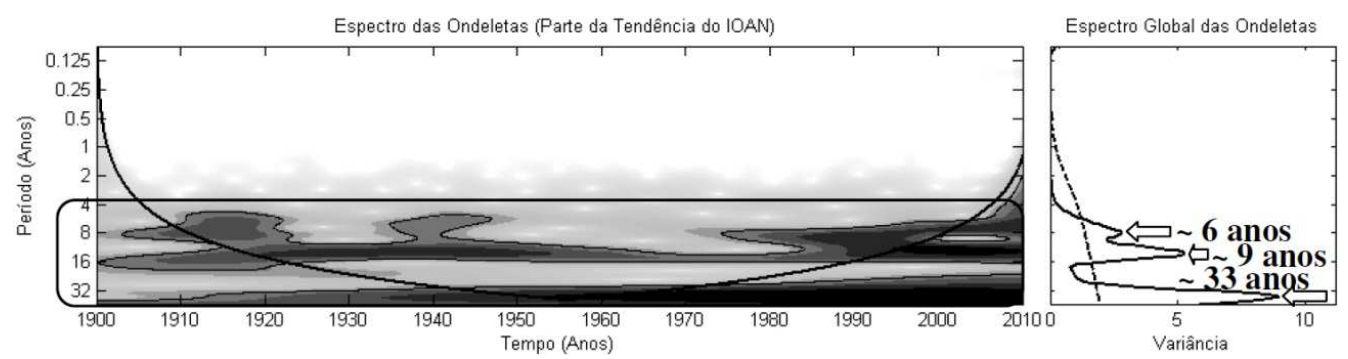

(a)

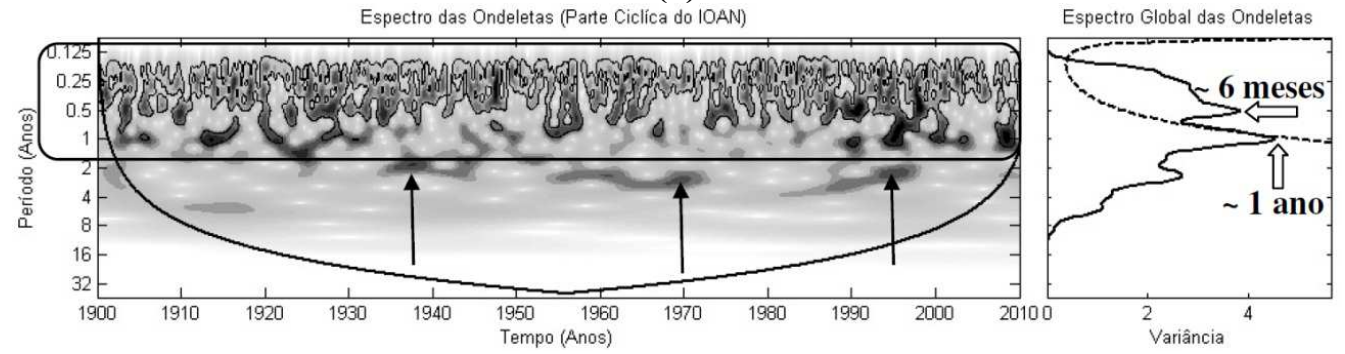

(b)

Figura 4: Análise das ondeletas aplicadas às séries (a) da tendência e (b) parte cíclica/aleatória, obtidas após tratamento da série dos índices de OAN com o filtro HP com os respectivos Espectro de Potência (EPO, esquerda) e Espectro Global das Ondeletas (EGO, direita).

\subsection{Relação dos índices com a variabilidade da precipitação no Brasil}

O objetivo de se estudar séries temporais de índices climáticos é procurar identificar possíveis ciclos ou periodicidades, que auxiliem na elaboração de previsão climática. O EGO da parte cíclica/aleatória do IOS (Figura $2 b$ ) mostra um ciclo de 0,5 anos (6 meses), com confiabilidade superior ao nível de $95 \%$, que está presente nessa série de dados mensais e ser composta de ciclos inferiores a seis anos. Esse ciclo, possivelmente relacionado com o aquecimento continental, foi utilizado para elaborar correlações lineares entre a série cíclica/aleatória do IOS e a precipitação, compilada e interpolada em pontos de grade $0.5^{\circ} \times 0,5^{\circ}$ pela UDEL, com o IOS adiantado de 1, 3 e 6 meses com relação à precipitação. Neste trabalho, só é mostrada a correlação entre a média de 6 meses da série cíclica/aleatória do IOS (IOS6, de abril a setembro, adiantado de 6 meses) e a precipitação média de outubro a março, época do ano em que, de maneira geral, chove mais em grande parte da região tropical do País. Gan et al. (2004) mostram que $90 \%$ da precipitação na região CentroOeste do Brasil ocorre entre Outubro e Abril. Na Figura 5a, é mostrado o mapa de isolinhas de coeficientes de correlação entre a série original do IOS6, sem filtragem, e a precipitação média de outubro a março para o intervalo 1948-1999, que engloba as fases fria e quente da ODP e os eventos El Niño/La Niña. $\mathrm{Na}$ Figura 5b, o mapa é semelhante ao anterior só que para a série cíclica/aleatória do IOS6, isto é, retirado o componente da tendência composta de ciclos superiores a 6 anos.
Nas duas figuras, os valores dos coeficientes de correlação apresentam módulos maiores no norte da Amazônia e no sudoeste do RS, com sinais contrários e núcleos centrais deslocados geograficamente. Todavia, quando se comparam os dois mapas, nota-se uma configuração distinta das isolinhas de coeficientes de correlação, particularmente nas áreas de transição climática entre as Regiões Norte e Nordeste e na área de atuação da ZCAS (Figura 5b). Com o $\mathrm{IOS}_{6}$ (previsor), notam-se valores altos de coeficiente de correlação ao Norte e ao Sul do Brasil, de até 0,72 e -0.67, respectivamente, com nível de confiança superior a $99 \%$, sugerindo dominância da circulação atmosférica de escala global nessas regiões. Entretanto, sobre o leste e sul da Amazônia e Centro-Oeste, os coeficientes alcançaram 0,2 , possivelmente devido ao posicionamento variável da ZCAS. Grimm (2003) mostra que a análise sazonal tende a filtrar as anomalias da circulação atmosférica e de precipitação de escala temporal menor, pois ocorrem mudanças abruptas durante as monções de verão, sugerindo que os processos regionais, em particular o aquecimento continental, dominem os de influência remota. No caso da região da ZCAS, por exemplo, existe pequeno impacto na escala sazonal, mas impacto significativo na intensificação da convecção em janeiro e seu decaimento em fevereiro nos eventos El Niño (Grimm, 2004). Embora esses valores de coeficiente de correlação sejam baixos, eles são confiáveis ao nível superior a $99 \%$, devido ao período de dados utilizado (52 anos). Aparentemente, a correlação com o $\mathrm{IOS}_{6}$ cíclico/aleatório melhora a análise com relação à série de IOS original, tanto nos módulos dos coeficientes de correlação quanto na configuração espacial de 


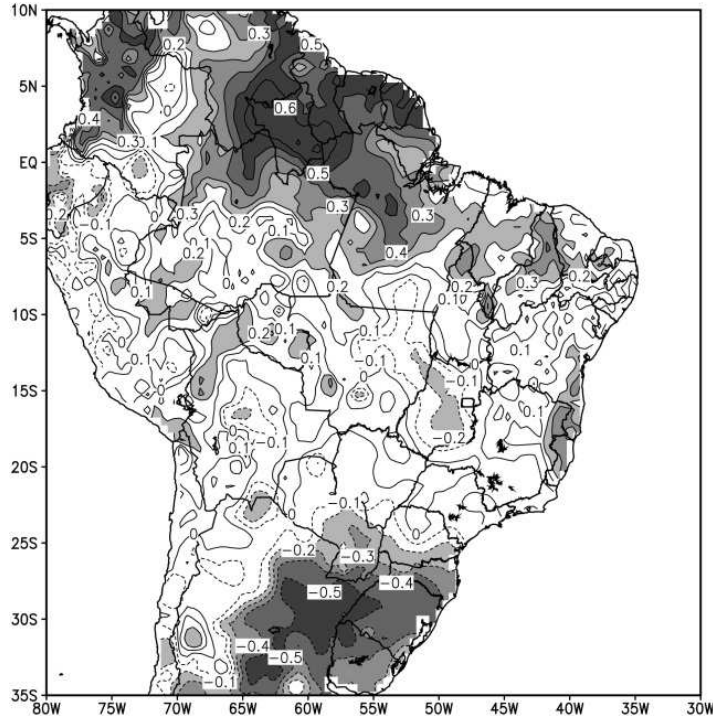

(a)

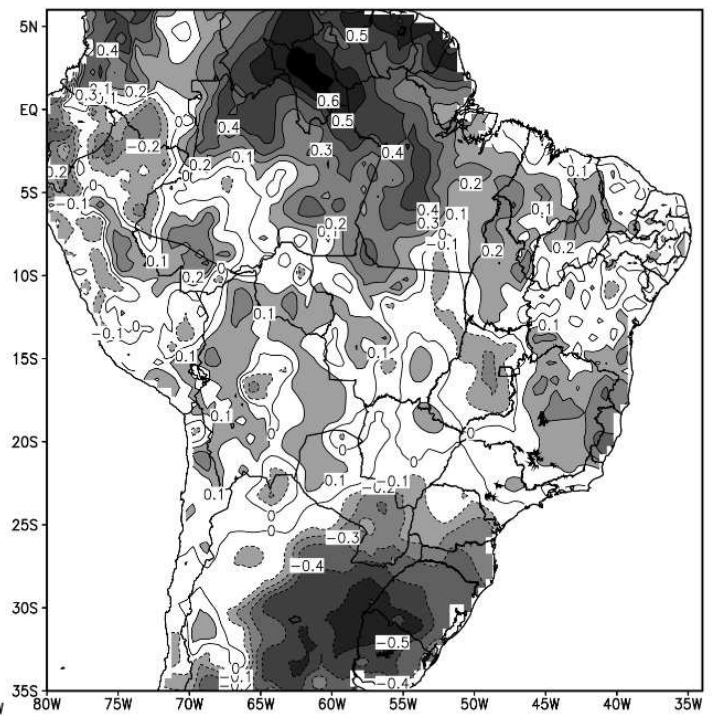

(b)

Figura 5: Mapas de coeficientes de correlação entre a precipitação interpolada (UDEL), média de outubro a março, e (a) a série do IOS6 original (sem filtragem), média de abril a setembro, e (b) a série cíclica/aleatória do IOS6, média de abril a setembro, da qual foi retirado o componente de tendência para o período 1948-1999.

Fonte dos dados: ESRL/PSD/NOAA.

suas isolinhas. O EGO da tendência do IOS (Figura 2a) indica a existência de periodicidades de 6, 9 e 18 anos e de 28 anos na série desse índice. Devido à alta correlação existente entre o IOS e a precipitação em algumas áreas do Brasil, talvez seja possível utilizar essas periodicidades para estimar a qualidade de uma estação chuvosa nessas áreas dentro desses intervalos de tempo. O pico de 6 anos parece estar relacionado com ciclos completos de eventos El Niño/La Niña e a sua influência na precipitação está amplamente documentada (e.g. Grimm et al, 2000; Grimm, 2003; Andreoli e Kayano, 2005). A título de teste, foi elaborada uma correlação (não mostrada) entre $\operatorname{IOS}_{6}$ e precipitação, semelhante à análise anterior, somente para o intervalo 1976-2005 em que predominaram IOS negativos (Figura 1a). Os valores dos coeficientes foram de até 0,84 e $-0,77$ no Norte e no Sul do Brasil, respectivamente, com nível de confiança superior a $99 \%$. Esses resultados sugerem que a circulação de grande escala seja o modo dominante no Norte e no Sul do Brasil, pois a OS sozinha explicou mais de $50 \%$ da variância da precipitação das regiões citadas e poderia ser usada como um estimador da qualidade da precipitação média de outubro a março, com até 6 meses de antecedência. Com IOS positivos (em geral, eventos La Niña), haveria precipitação acima (abaixo) da média no Norte (Sul) do País. Para IOS negativos (possivelmente eventos El Niño), a configuração da precipitação seria oposta. Porém, deve se levar em conta, como alerta Grimm (2003), que a variabilidade intrasazonal é grande devido à predominância dos fenômenos regionais sobre os de escala planetária no auge do verão.
Usou-se o mesmo procedimento acima para o Índice da Oscilação do Atlântico Norte (IOAN). Na Figura 6a, é mostrado o mapa de correlação linear entre a série do IOAN6 original, sem filtragem, e a precipitação para o período que engloba as fases fria e quente da ODP (1948-1999). O IOAN6 é a média desse índice de abril a setembro, adiantado de 6 meses (previsor) com relação à precipitação média de outubro a março. Na Figura 6b, o mapa é semelhante ao anterior só que para a série cíclica/ aleatória do IOAN6, isto é, retirado o componente da tendência.

De maneira geral, os módulos dos coeficientes de correlação foram inferiores a 0,2 , não atingindo nível de confiança estatística, exceto nas regiões norte da Amazônia e no Centro-Leste do Brasil em que os valores atingiram - 0,43 e 0,52, respectivamente, sendo os valores de módulo maior relativos à serie IOAN6 original (Figura 6a), em que o nível de confiança foi superior a $99 \%$. Quando a análise foi elaborada apenas para o período 1976-1999, correspondente à fase quente da ODP (figura não mostrada), os coeficientes de correlação entre a precipitação e os índices de OAN alcançaram valores de até $-0,55$ no norte e 0,69 no centro-leste do Brasil. Aparentemente, na maior parte do Brasil, o índice de OAN não é adequado para a previsão de precipitação como ocorreu com o IOS. Em princípio, isso já era esperado pela própria definição dos índices da OAN, cuja atuação primordial é no norte do Atlântico Norte, fora da região tropical. Como a região Centro-Leste apresentou nível de confiança elevado, sugere-se um estudo mais aprofundado para se identificar um possível mecanismo físico que possa relacionar a chuva regional à $\mathrm{OAN}$. 


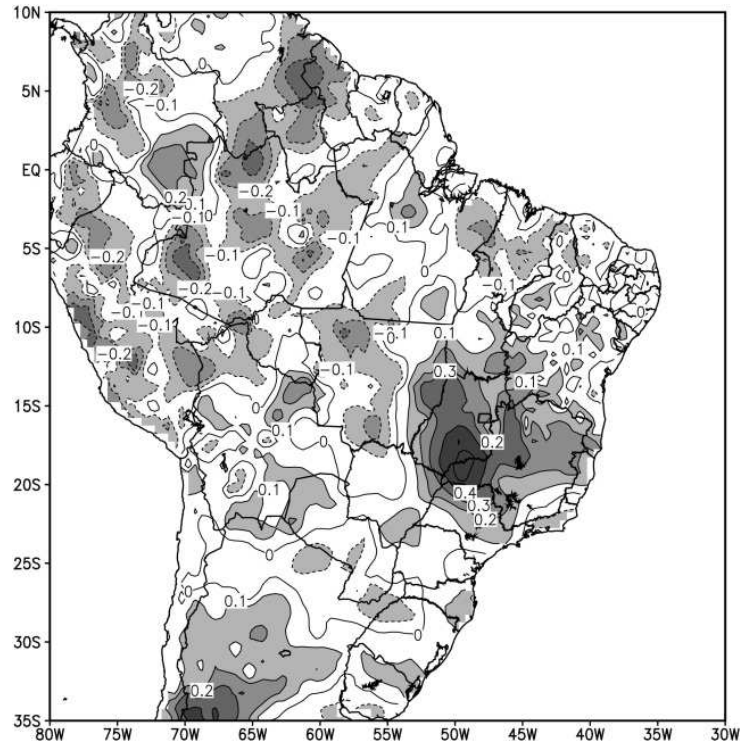

(a)

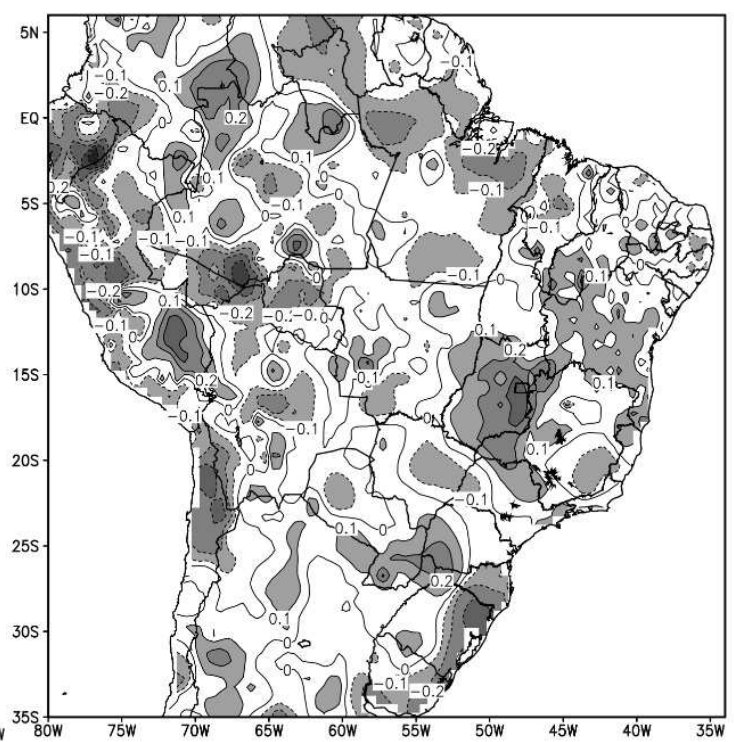

(b)

Figura 6: Igual à Figura 5, porém para a OAN.

Fonte dos dados: ESRL/PSD/NOAA.

Resultados, semelhantes aos aqui apresentados, foram publicados, porém utilizando índices oceânicos e não atmosféricos. Grimm (2004) usa dados de TSM Niño 3 e dados de reanálises NCAR/NCEP para mostrar a influência dos eventos La Niña nas monções de verão no Brasil. Andreoli e Kayano (2005), utilizando os índices de TSM Niño3, elaboram estudos diagnósticos para a América do Sul e mostram que os impactos dos eventos El Niño/La Niña são dependentes das fases da ODP. O número de eventos La Niña da fase quente da ODP foi baixo, dois apenas, não permitindo que fossem feitos testes de significância estatística. Contudo, as análises da precipitação mostram que esses dois eventos produziram totais pluviométricos muito elevados na Amazônia e Nordeste do Brasil. Os índices de TSM Niño 3, estratificados de acordo com as fases da ODP, foram usados por Kayano e Andreoli (2006), que concluem que as configurações da precipitação na Bacia do Prata são robustas e bem definidas, quando ENOS e ODP estão na mesma fase. Alertam, ainda, que esses aspectos devem ser levados em consideração no monitoramento e na previsão climática. Além disso, Kayano e Andreoli (2007), utilizando índices de TSM Niño 3.4 e agrupando os índices de OPD em três seções, altos, baixos e neutros, exploram os impactos dos eventos ENOS na precipitação da América do Sul.

\section{SUMÁRIO E CONCLUSÃO}

O objetivo deste artigo é introduzir uma técnica de filtragem de séries temporais que é amplamente utilizada em Econometria, mas, pelo conhecimento dos autores, não é em análise de séries temporais climáticas. A técnica de filtragem Hodrick-Prescott, associada à das ondeletas de Morlet, mostra ser eficiente na separação do sinal da tendência (baixas frequências) com relação aos sinais cíclicos/aleatórios (altas frequências). O problema crucial da aplicação do filtro HP é a escolha do valor do parâmetro de penalização $(\lambda)$ utilizado que, desde sua origem, é arbitrária. Neste trabalho preliminar, é testado apenas o valor $\lambda=14.400$ para análise de séries de dados médios mensais dentre os citados na literatura. Esse valor mantem os ciclos de períodos superiores a 6 anos na série da tendência e os ciclos inferiores são deslocados para a série cíclica/aleatória.

$\mathrm{Na}$ série do Índice de Oscilação Sul, a série da tendência mostra um modo essencialmente positivo da OS entre 1917 e 1976, exceto entre os anos 1939/1942, em que ocorreu um evento El Niño forte. Entre 1977 e 2005, o modo da OS é predominantemente negativo, porém os eventos La Niña fortes de 1988/1989 e 1998/2001 são aparentes. Nesse período, a amplitude do sinal também é maior, indicando um modo ou estado climático com grande variabilidade interanual, comparado com o do período anterior. Além do ciclo de 6 anos relativo ao Ciclo El Niño/La Niña, destacam-se os ciclos de 9, 17 e 28 anos, os dois primeiros possivelmente relacionados ao Ciclo Nodal Lunar (Ciclo Saros) e, o último, às fases da ODP, que pode ser um múltiplo do Ciclo Saros (Yndestad, 2006). Na análise dos índices de OAN, a série da tendência não apresenta amplitudes significativas, sugerindo que as frequências da OAN sejam naturalmente altas, ou seja, um sinal de grande variabilidade temporal. Entretanto, o espectro de potência 
identifica ciclos de 6, 9 e 33 anos bem definidos na série da tendência, sugerindo associação com o Ciclo de El Niño/La Ninã, Ciclo Saros ( 9 anos) e seu possível múltiplo de 36 anos.

As correlações lineares da precipitação com o IOS e os índices de OAN para o Brasil, mostram que a OS apresenta um potencial melhor que a OAN como estimador da qualidade da precipitação, particularmente para o norte do continente sul-americano. Para o período 1948-1999, os valores dos coeficientes de correlação do IOS6 com a precipitação média observada na estação chuvosa (outubro-março) são de até 0,72 e -0.67 para o Norte e para o Sul do país, respectivamente, com nível de confiança superior a $99 \%$. Os valores chegam até 0,84 (Norte) e - 0,77 (Sul) para o período recente de 19772005 , em que predominaram IOS negativos durante 28 anos, associados à fase quente da ODP em princípio. $\mathrm{Na}$ fase fria anterior da ODP, os IOS foram predominantemente positivos e a frequência de eventos La Niña foi maior do que a de El Niño, tendo sido estes, em geral, fracos e moderados e de baixa duração, inferior a 10 meses. Considerando que a ODP entrou em uma nova fase fria, possivelmente a partir de 1999, e nela poderá permanecer por 28 anos, ou seja, até cerca de 2030. Portanto, sugere-se que a previsão da precipitação possa ser feita utilizando os dados do IOS monitorados no período anterior em que permaneceram negativos entre 1946-1976. Sua média a cada 3 (IOS3) e/ou 6 meses (IOS6) seria utilizada na previsão qualitativa da estação chuvosa, com 3 e/ou 6 meses de antecedência.

\section{AGRADECIMENTOS}

Os autores agradecem a Profa. Dra. Alice M. Grimm pela valiosa contribuição na revisão e sugestões para implementação no presente artigo.

\section{REFERÊNCIAS BIBLIOGRÁFICAS}

ANDREOLI, R.V.; KAYANO, M.T. ENSO-related rainfall anomalies in South America and associated circulation features during warm and cold Pacific Decadal Oscillation regimes. International Journal of Climatology, v. 25, p.2017-2030, 2005.

DAUBECHIES, I. Ten Lectures on Wavelets. SIAM, 357 p, 1992.

FOUFOULA-GEORGIOU, E.; KUMAR, P. Wavelets in Geophysics: Wavelet analysis and its applications. San Diego, Academic Press, 372p, 1994.

GABOR, D. Theory of Communication. Journal of the Institution of Electrical Engineers, v. 93,p. 429-457, 1946.

GAN M. A., KOUSKY, V. E. , ROPELEWSKI, C. The South America monsoon circulation and its relationship to rainfall over West-Central Brazil. Journal of Climate, v. 17, n. 1, p 47-66, 2004.

GRIMM, A.M.; BARROS, V.R.; DOYLE, M.E. Climate variability over Southern South America associated with El Niño and La Niña events. Journal of Climate, v. 13, p. 35-58, 2000.

GRIMM,A.M.TheElNiñoimpactonthesummermonsooninBrazil: regional processes versus remote influences. Journal of Climate, v. 16, p. 263-280, 2003

GRIMM, A.M. How do La Niña events disturb summer monsoon system in Brazil? Climate Dynamics, v. 16, p. 123-138, 2004.

GROSSMAN, A; MORLET, J. Decomposition of Hardy functions into square integrable wavelets of constant shape. Society for Industrial and Applied Mathematics Journal on Mathematical Analysis, v. 15, p. 732-736, 1984.

HODRICK, R. J.; PRESCOTT, E.C. Postwar U.S. Business Cycles: an Empirical Investigation; mss. Pittsburgh: Carnegie-Mellon University: Discussion Paper 451, Northwestern University, 1980.

JONES, P.D., JÓNSSON, T.; WHEELER, D. Extension to the North Atlantic Oscillation using early instrumental pressure observations from Gibraltar and South-West Iceland. International Journal of Climatology, v. 17, p.1433-1450, 1997.

KAYANO, M. T.; ANDREOLI, R.V. Low frequency variability. In: Climate Change in the La Plata Basin, Chapter XIV, Ed. Barros, V.; Clarke, R.; Silva Dias, P., p.183-198, CIMA, UBA, ISBN 950-692-066-4, 2006.

KAYANO, M.T; ANDREOLI, R.V. Relations of South American summer rainfall interannual variations with the Pacific Decadal Oscillation. International Journal of Climatology, v. 27, p.531-540, 2007.

KING, R. G.; REBELO, S.T. Low frequency filtering and real business cycles. Journal of Economic Dynamics and Control, v. 17, n. 1,2, p. 207-231, 1993.

MAKSIMOV, I.V.; SMIRNOV, N.P. A contribution to the study of causes of long-period variations in the activity of the Gulf Stream. Oceanology, v. 5, n. 2, p.15-24 (Tradução da American Geophysical Union do original em Russo), 1965.

MARAVALL, A.; RIO, A. Time aggregation and the HodrickPrescott filter. Servicio de Estudios, Documento de Trabajo $\mathbf{n}^{\circ}$ 0108, 43p., Banco de España, 2001.

MOLION, L.C.B. Aquecimento global, El Niños, Manchas Solares, Vulcões e Oscilação Decadal do Pacífico. Climanalise, agosto, CPTEC/INPE, 2005, disponível em: http://www.cptec.inpe.br/products/climanalise/artigos/ molion0805.pdf

MORLET, G. A.; FOURGEAU, I.; GIARD, D. Wave Propagation and Sampling Theory. Part 1: Complex Signal 
and Scattering in Multilayered Media. Geophysics, v. 47, n. 2, p. 203-221, 1982.

OSBORN, T. J.; BRIFFA, K.R.; TETT,S.F.; JONES, P.D.; TRIGO, R.M.. Valuation of the North Atlantic Oscillation as simulated by a climate model. Climate Dynamics, v. 15, p. 685- 702, 1999.

R DEVELOPMENT CORE TEAM R: A language and environment for statistical computing. R Foundation for Statistical Computing, Vienna, Austria, ISBN 3-90005107-0, 2011, disponível em: http://www.R-project.org/

RAVN, M.O. ; UHLIG, H. On adjusting the Hodrick-Prescott filter for the frequency of observations. The Review of Economics and Statistics, v. 84, n. 2, p.371-380, 2002.

RASMUSSON, E. M.; CARPENTER, T. H. Variations in tropical sea surface temperature and surface wind fields associated with the Southern Oscillation/ El Niño. Monthly Weather Review, v. 110, p. 354-384, 1982.
TORRENCE, C.; COMPO, G.P. A practical guide to wavelet analysis. Bulletin of American Meteorological Society, v.79, . 1, p.61-78, 1998.

TRENBERTH, K. E. The Definition of El Niño. Bulletin of American Meteorological Society, v. 78, n. 12, p. 271277, 1997.

TROUP, A.J. The southern oscillation. Quarterly Journal of the Royal Meteorological Society, v. 102, p.490-506, 1965.

YNDESTAD, H. The influence of the nodal cycle on Arctic climate. ICES Journal of Marine Science, v. 63, p.401420, 2006.

WENG, H.; LAU, K.M. Wavelet, period doubling, and timefrequency localization with application to organization of convection over the Tropical Western Pacific. Journal of Atmospheric Sciences, v. 51, p.2523-2541, 1994. 